\title{
Criminologie
}

\section{La couverture de presse et ses fonctions légitimantes}

\section{Ronald D. Crelinsten}

Volume 20, numéro 1, 1987

Faire les nouvelles : journalisme et affaires criminelles

URI : https://id.erudit.org/iderudit/017245ar

DOI : https://doi.org/10.7202/017245ar

Aller au sommaire du numéro

Éditeur(s)

Les Presses de l'Université de Montréal

ISSN

0316-0041 (imprimé)

1492-1367 (numérique)

Découvrir la revue

\section{Citer cet article}

Crelinsten, R. D. (1987). La couverture de presse et ses fonctions légitimantes. Criminologie, 20(1), 35-57. https://doi.org/10.7202/017245ar

\section{Résumé de l'article}

This paper examines the legitimating function of press coverage by means of a specific case study of political violence. Dissident actors who resort to violence to achieve their political goals are generally treated by the authorities as common criminals. This criminal justice model is reproduced and reinforced in the press by selective focus on specific, narrow topics at the expense of political analysis. These topics include the victims, the threat of future attacks, police activity, and the declarations of those in authority. In the October Crisis of 1970, this process was temporarily disrupted and a transient symmetry was achieved whereby the point of view of the dissident actors and their supporters received as much attention as the official perspective of the authorities. With the invocation of the War Measures Act, this symmetry was destroyed and press coverage once again returned to an almost exclusive focus on official definitions of the situation. The author suggests that this pattern of press coverage reflects a transient disruption in the legitimating function of the media whereby, in normal times, the reporting of "news" can reproduce and reinforce official views of dissident actors who use violence for political ends.
Ce document est protégé par la loi sur le droit d'auteur. L’utilisation des services d’Érudit (y compris la reproduction) est assujettie à sa politique d'utilisation que vous pouvez consulter en ligne.

https://apropos.erudit.org/fr/usagers/politique-dutilisation/ 
This paper examines the legitimating function of press coverage by means of a specific case study of political violence. Dissident actors who resort to violence to achieve their political goals are generally treated by the authorities as common criminals. This criminal justice model is reproduced and reinforced in the press by selective focus on specific, narrow topics at the expense of political analysis. These topics include the victims, the threat of future attacks, police activity, and the declarations of those in authority. In the October Crisis of 1970 , this process was temporarily disrupted and a transient symmetry was achieved whereby the point of view of the dissident actors and their supporters received as much attention as the official perspective of the authorities. With the invocation of the War Measures Act, this symmetry was destroyed and press coverage once again returned to an almost exclusive focus on official definitions of the situation. The author suggests that this pattern of press coverage reflects a transient disruption in the legitimating function of the media whereby, in normal times, the reporting of "news" can reproduce and reinforce official views of dissident actors who use violence for political ends.

\section{INTRODUCTION}

Aujourd'hui, les hommes politiques et les autorités policières questionnent sérieusement le rôle que jouent les médias d'information dans la couverture des événements terroristes et des prises d'otages. Les médias, eux-mêmes, s'interrogent à ce sujet. Le détournement du vol 847 de la Trans-World Airlines (TWA) en juin 1984, l'assaut des Arméniens contre l'ambassade turque à Ottawa en mars 1985, la prise d'otage au consulat des Bahamas à Ottawa en avril 1986, constituent quelques exemples des récents incidents à l'origine de ces questionnements. Le problème se présente sous différentes formes - comme le danger des reportages sur les activités policières lors d'une prise d'otage, ou les interviews en direct avec les terroristes ou les ravisseurs - mais une des questions centrales reste dans quelle mesure la couverture des incidents terroristes confere-t-elle aux acteurs un degré de légitimité qu'ils ne pourraient jamais obtenir autrement. Par exemple, l'interview accordée le 5 mai 1986 par la National Broadcasting Corporation (NBC) à Abul Abbas, l'inspirateur présumé de l'attentat contre le navire Achille-

\footnotetext{
*Ronald D. Crelinsten, professeur adjoint, Département de criminologie, Université d'Ottawa.
} 
Lauro, fut sévèrement critiqué par le gouvernement Reagan, non seulement parce que le poste de télévision n'a pas divulgué le lieu où cette interview s'est déroulée, mais aussi parce que l'interview a permis au leader palestinien d'exposer ses idées au public américain. Pour certains membres du gouvernement américain, c'était accorder la légitimité à un criminel responsable de la mort d'un citoyen américain, donc légitimer un assassin. Mais est-il vrai que la couverture par la presse accorde nécessairement la légitimité à ceux qui deviennent l'objet de ces reportages? Est-ce que vraiment et de façon systématique la presse peut se permettre de légitimer un assassin ou des groupes minoritaires ou dissidents? Peut-elle le faire durant une longue période et à quelles conditions? Ou, est-ce que la presse se doit éventuellement de renforcer les vues officielles plutôt que celles des dissidents?

Dans cet article, j'examinerai ces questions à travers la couverture d'un incident spécifique remontant à seize ans déjà, soit la crise d'octobre 1970. Déjà à l'époque, cette crise politique soulevait beaucoup des questions qui se posent maintenant sur le rôle des médias d'information dans la légitimation des gens dits terroristes. De nos jours, c'est la télévision qui fait l'objet central des discussions brûlantes sur le rôle légitimant des médias. Dans la crise d'octobre, c'était plutôt la radio qui occupait la première place, quelques postes servant de "boîtes postales" pour les communiqués des ravisseurs. En dépit de la préséance des médias électroniques dans la diffusion des nouvelles dans notre "village global", la presse écrite a toujours joué un rôle déterminant dans la présentation, la structuration et, surtout, l'analyse des nouvelles. Cet article mettra donc l'accent sur la presse écrite, même si la radio et la télévision mériteraient un traitement semblable. Aussi nous nous limiterons à la légitimation telle qu'elle apparaît dans les reportages eux-mêmes. Quand on parle de légitimation, on ne peut parler que de celle qui se reflète dans les images de la presse écrite. Pour parler vraiment d'une légitimation au sein du public, il faudrait mesurer l'impact de la couverture de presse sur l'opinion publique.

\section{VIOLENCE, TERRORISME ET COMMUNICATION}

Pour ceux qui ne possèdent pas de pouvoir, être reconnus comme acteurs légitimes dans la vie politique n'est pas chose facile. Pour les promoteurs d'un changement social radical, le problème est d'autant plus aigu que beaucoup de voies légitimées, telles que celles des partis politiques traditionnels, sont fermées aux idées radicales. Qui plus est, les moyens traditionnels de protestation, comme la manifestation publique, sont généralement réprimés et criminalisés au moment où ils attei- 
gnent un certain succès. Or, la violence se présente souvent comme une solution - au moins à court terme - pour capter l'attention des gens au pouvoir ou du grand public. Les actions violentes - une prise d'otage ou un attentat à la bombe - qui se situent dans un contexte politique, constituent un moyen, pour l'acteur politique radical ou marginal, d'attirer l'attention des personnes cibles. Vu de cette façon, la violence d'inspiration politique peut être conçue comme un moyen de communication (cf. Schmid et de Graaf, 1982). Dans une stratégie terroriste, la violence joue un rôle plus particulier encore. De par la menace et l'utilisation de la violence contre un groupe de personnes ou d'objets cibles, l'acteur politique essaie de contraindre un autre groupe de personnes, généralement les gens au pouvoir ou ceux qui peuvent les influencer, à répondre à ses revendications politiques.

Quatre principaux dangers peuvent faire échouer cette stratégie. En premier lieu, les autorités et autres audiences publiques ne pourraient accorder leur attention qu'à l'acte de violence, en ignorant totalement les buts politiques dont il n'est que le véhicule. En effet, l'utilisation de la violence par les acteurs politiques radicaux peut servir aux autorités de prêtexte pour les marginaliser plus encore. Ceci nous amène au deuxième danger qui menace l'utilisation de la violence dans un contexte politique. Aussitôt qu'un acteur politique agit en dehors des institutions légitimées et adopte la violence, le processus pénal se déclenche, entraînant généralement la répression policière. Dans les pays de tradition anglo-saxonne, le crime politique n'a pas d'existence en tant que tel (cf. Allen, 1974). Toute action politique qui enfreint la loi est traitée comme un crime de droit commun. Ce qui a pour fonction de stigmatiser l'action politique dissidente et de saper toutes ses chances de légitimité aux yeux du grand public.

Ceci nous amène au troisième grand danger associé à la violence d'inspiration politique: la réprobation du public et, surtout, des sympathisants de la cause politique revendiquée par l'acteur violent. L'utilisation de la violence peut briser les liens de solidarité existant entre divers groupes qui partagent les mêmes buts. Le quatrième danger, finalement, est la façon dont l'action terroriste ou violente est traitée par les médias. Si l'on place au premier plan les victimes et leurs familles, si l'on insiste sur l'aspect violent en soi, et si l'on ignore l'élément politique de l'action et ses antécédants, la couverture de l'incident peut aller à l'encontre du but poursuivi par ses auteurs. L'image criminelle de l'action promulguée par la réaction des autorités peut aussi être renforcée par un reportage sensationnaliste. Dans un tel cas, le message politique pourrait être contrecarré par l'attention sélective sur la violence. 
En effet, les recherches sur la manière dont les médias d'information traitent les incidents dits terroristes démontrent que les actions violentes attirent facilement l'attention des médias et de leurs publics, et obtiennent pour leurs auteurs la publicité auprès du grand public. Cependant, il est rare que la couverture de presse soit telle que les auteurs gagnent l'approbation de leurs actes et, donc, la légitimité (Kelly et Mitchell, 1981; voir aussi Halloran, 1981: 133-135).

\section{LA CRISE D'OCTOBRE COMME CRISE DE LÉGITIMATION}

Dans une étude de cas en profondeur des quinze premiers jours de la crise d'octobre, j'ai démontré que les autorités ont tenté de dépeindre l'action du F.L.Q. comme une affaire criminelle tout au long de la période précédant l'invocation des mesures de guerre (Crelinsten, 1985). Cependant, grâce à une manipulation habile des médias d'information par les ravisseurs de James Cross, la diffusion constante et fidèle de leurs communiqués dans les journaux et à la radio fut assurée dans la première semaine de la crise et le manifeste du F.L.Q. fut diffusé par Radio-Canada. Ceci eut comme résultat que les paroles du F.L.Q. ont pénétré le réseau de communication pour contrecarrer ce «modèle pénal» qui traite l'acte terroriste d'acte criminel plutôt que d'acte politique.

Bien que la plupart des gens dits ordinaires n'approuvaient pas l'action du F.L.Q. en tant que telle, ils ont exprimé un degré de sympathie fort élevé pour l'esprit du manifeste et pour la vision de la vie politique, sociale et économique au Québec qui s'y trouve. Cette sympathie a étonné et déconcerté les autorités et a induit Jérôme Choquette, ministre de la Justice du Québec, à adopter un ton conciliant en livrant l'offre finale aux ravisseurs de James Cross, malgré une insistance de la part d'Ottawa pour ne rien concéder sur le plan de la libération des prisonniers felquistes.

Dans le sillage de l'enlèvement de Pierre Laporte et de l'escalade du débat public sur le statut des négociations entamées par le gouvernement Bourassa avec les deux cellules felquistes, la dimension politique de l'affaire a pris le pas sur le sort des otages et les demandes spécifiques des ravisseurs. Quand le débat s'est généralisé au point que le Premier ministre de l'Ontario déclare qu'une "guerre générale» existe au Québec, que seize leaders québécois provenant des milieux intellectuel, syndical, financier et péquiste se disent en faveur des négociations (la déclaration des seize sous l'impulsion de René Lévesque et Claude Ryan) et que les étudiants universitaires amorcent une opération de débrayage en faveur du F.L.Q., le modèle de guerre a remplacé le modèle pénal, l'armée fut appelée et la loi sur les mesures de guerre, invoquée. Dans l'étude de cas 
citée plus haut, je démontre que cette transition reflète une crise de légitimation dans laquelle le moyen traditionnel (le modèle pénal) pour stigmatiser la violence d'inspiration politique ayant échoué, on a eu recours à un moyen extraordinaire (le modèle de guerre) pour accomplir la même tâche, à savoir de "dépolitiser» en criminalisant (Crelinsten, 1985, partie VI).

En deuxième partie, nous examinerons la couverture par la presse des événements au cours de cette période de quinze jours afin de découvrir comment cette lutte autour de la légitimation se reflète dans la manière dont les événements furent rapportés par la presse écrite. La couverture de presse, a-t-elle réussi à légitimer, pour une brève période, l'action des cellules felquistes ou a-t-elle plutôt renforcé les vues officielles provenant des gouvernements? Nous utiliserons quelques techniques de reportage pour illustrer notre analyse: la sélection des manchettes, surtout à la une, le choix des photos, également à la une, la composition d'une page, et la situation des reportages dans le journal. Notre échantillon pour cette analyse se compose de six quotidiens montréalais dont quatre de langue française et deux de langue anglaise, de même qu'un quotidien de Québec, le Soleil. Nous les classons approximativement ici, selon un ordre basé sur l'heure de leur publication: le Devoir, The Gazette, le Journal de Montréal, Montréal-Matin, la Presse, le Soleil, et The Montreal Star. Soulignons que le Journal de Montréal et Montréal-Matin, étant des journaux de petit format, ne portent que les manchettes et les photos à la une.

\section{LES NOUVELLES, UNE CONSTRUCTION SOCIALE QUI REPRODUIT L'ORDRE}

Plusieurs chercheurs intéressés aux médias démontrent que «les nouvelles" ne présentent pas la réalité objective, mais sont plutôt la représentation d'une réalité construite selon un processus de sélection, d'interprétation et de présentation (Tuchman, 1978; Cohen et Young, 1981). Qui plus est, à travers ce processus de construction sociale, les médias reproduisent les idéologies dominantes puisque leurs règles institutionnelles reflètent et renforcent les mêmes structures sociales qui informent leurs reportages (voir Hall et al., 1978; Molotch et Lester, 1974; Murdock, 1981). En conséquence, la perspective officielle des événements est reproduite et les perspectives alternatives sont systématiquement écartées ou minimisées (Hall et al., 1978; Schlesinger $e t$ al., 1983). L'utilisation des sources officielles d'information, telles que la police, les politiciens, les bureaucrates, les porte-parole des institutions légitimées, constitue un des principaux moyens pour accomplir cette 
reproduction de l'ordre. Stuart Hall et ses collègues établissent une distinction entre les sources primaires et les sources secondaires (primary definers et secondary definers). Les sources primaires sont des individus occupant des postes d'autorité, qui rapportent les événements selon la perspective officielle: un acteur politique qui utilise la violence est un criminel. Les sources secondaires sont des individus extérieurs à la structure de pouvoir, qui rapportent les événements selon des perspectives différentes et souvent opposées à la perspective officielle: vu l'oppression politique, la violence est justifiée ou, du moins, compréhensible. Ces chercheurs notent aussi que les sources primaires servent à établir le champ de discussion dans lequel les sources secondaires doivent présenter les définitions alternatives, même si elles réussissent à pénétrer les reportages. Ce processus de l'établissement de l'ordre du jour (agenda-setting) rend, de ce fait, toutes les perspectives alternatives hors d'ordre (Hall et al., 1978: 57-60).

Dès le début de la crise d'octobre, soit le 6 octobre, le lendemain de l'enlèvement de James Richard Cross, la victime elle-même (l'otage), la menace de mort qui pèse sur elle, les délais imposés par ses ravisseurs, et l'identité de ces derniers sont les principaux thèmes à la une des quotidiens. La même chose se répétera lors du rapt de Pierre Laporte, cinq jours plus tard. L'accent mis sur les victimes se reflète dans les photos à la une. M. Cross ou son entourage figurent sur seize des vingt-trois photos à la une portant sur l'affaire Cross pendant les trois premiers jours de la crise. M. Laporte ou les membres de sa famille (sa femme ou sa mère) apparaissent sur cinq des sept photos à la une des journaux qui publient pour la première fois après son enlèvement'. $M$. Cross figure sur une sixième. Parmi cent-onze photos publiées à la une avant la mort de Pierre Laporte, le 18 octobre, 41 (soit $37 \%$ ) montrent les victimes, leurs résidences, leur entourage.

L'emphase sur les victimes des deux rapts renforce l'image officielle selon laquelle ces actes sont d'ordre criminel plutôt que politique. Cette image se traduit par des reportages sur les déclarations des hommes politiques, tel que Mitchell Sharp, ministre des Affaires étrangères, ou Jérôme Choquette, ministre québécois de la Justice, et sur l'enquête policière. Le fait qu'on insiste sur les menaces et les délais renforce l'aspect dramatique et extraordinaire de l'affaire. Par le fait même, l'événement est traité hors de son contexte social et politique. Donc, on voit mais on ne comprend pas. Voici quelques exemples de la façon dont les journaux peuvent renforcer le thème de menace, ce qui rend plus facile la construction d'une image criminelle.

1. M. Laporte fut enlevé un samedi soir, le 10 octobre, et seulement cinq des journaux étudiés publièrent le 12 octobre, lundi, jour de l'Action de Grâce. 
1. L'assassinat sélectif: Le lendemain du rapt de M. Cross, Marcel Dupré, correspondant parlementaire à Québec pour la Presse, parle d'une liste de cibles potentielles pour un assassinat sélectif, portant MM. Trudeau, Bourassa et Drapeau en tête. Le titre flamboyant de deux lignes à la une: «Bourassa, Trudeau et Drapeau sont sur la liste des personnes visées par le FLQ" (Dupré, la Presse, 6 octobre: A1) ${ }^{2}$. Il s'agit d'une fausse rumeur émanant d'une politique officielle de silence sur les vrais contenus du manifeste du F.L.Q.

2. Vol d'armes: Le 7 octobre, les deux journaux de l'après-midi publient à la une la nouvelle d'un vol de fusils sur un navire japonais à Trois-Rivières (Hardy, le Soleil, 7 octobre: 1-2; The Montreal Star, 7 octobre: 1). Tandis que le Star se limite aux détails du vol, Hardy, du Soleil, affirme que les autorités policières se demandent s'il s'agit d'un complot felquiste. En reliant entre-eux des événements disparates ${ }^{3}$, les journaux renforcent l'idée d'une menace d'envergure qui existerait d'ailleurs même sans les liens explicites établis par Hardy à l'aide de ses sources policières. L'article de The Montreal Star, intitulé "Cargaison de fusils volée" (traduction), est encadré et le texte imprimé en caractères un peu plus foncés que ceux des articles qui l'entourent, et qui tous portent sur l'affaire Cross.

3. La "prédiction" de Lucien Saulnier: Le 12 octobre, après l'enlèvement de $\mathrm{M}$. Laporte, plusieurs joumaux font référence aux événements du 7 octobre de l'année précédente, liés à la grève de la police de Montréal (Masson, la Presse, 12 octobre: B 1; The Montreal Star, 12 octobre: 2). Ils rappellent à leurs lecteurs l'avertissement passé de Lucien Saulnier, à savoir qu'une révolution se mijote au Canada. Dansla Presse, à la page $\mathrm{Bl}$, on trouve une photo de M. Saulnier, à l'époque, levant l'index comme pour dire, "Vous verrez bien, un jour..." La manchette de l'article accompagnant cette photo affirme: "Saulnier avait vu juste au même moment... il y a un an" ${ }^{4}$. Marcel Desjardins de la Presse (12 octobre: B7) et James Ferrabee de The Gazette (12 octobre: 5) font référence, tous les deux, au témoignage de $\mathrm{M}$. Saulnier lors de l'enquête parlementaire sur la Compagnie des Jeunes Canadiens où il parle de l'existence d'un plan révolutionnaire. L'impact de ce reportage en 1970 ,

2. Nous ne mentionnerons que le jour et le mois dans les citations des quotidiens. L'année est 1970, à moins d'avis contraire.

3. Beaucoup plus tard, on a découvert que le vol s'insère dans une lutte syndicale purement locale (Haggart et Golden, 1979: 9-10; Provencher, 1974: 14).

4. Le 1 l octobre 1969 à 18 h 00, M. Saulnier a convoqué une conférence de presse dans laquelle il réclame une enquête publique sur les activités dites subversives de la Compagnie des Jeunes Canadiens. Il y a eu, effectivement, une enquête parlementaire un mois plus tard. 
le choix des manchettes, et la composition des pages où figurent ces articles, renforcent l'idée qu'un deuxième enlèvement confirme les prévisions de M. Saulnier. Son avertissement de l'année précédente se change en prédiction, lors de l'escalade foudroyante que représente l'enlèvement de Pierre Laporte. On voit comment on construit une réalité par la juxtaposition d'images et des déclarations d'une source primaire. Le passé, transposé dans le présent, transforme l'image purement criminelle de la première semaine - avant l'enlèvement de M. Laporte - en image révolutionnaire. De cette façon, on préconise le passage d'un modèle de contrôle pénal à un modèle militaire. L'éditorialiste de $l a$ Presse, Jean-Paul Desbiens, accentue encore l'impression qu'on a atteint un point culminant. Sous le titre manichéen de «L'anarchie ou démocratie", il écrit: «il n'y a de choix qu'entre l'autorité de la loi et le règne de la terreur. Il faut s'atteler tout de suite à la consolidation de l'autorité démocratique (Desbiens, la Presse, 12 octobre: A4).

4. La déclaration d'une source primaire: Notons, finalement, que, lors de sa première interview, le 5 octobre, à peine quelques heures après l'enlèvement de $M$. Cross, le ministre des Affaires étrangères, Mitchell Sharp, déclare à Ottawa: "Nous espérons ardemment que les criminels seront arrêtés" (Turcotte, la Presse, 6 octobre: 6, souligné par nous).

\section{LE TRAITEMENT DES MOTS DU F.L.Q.}

Les ravisseurs de James Cross ont réussi, dès le troisième jour de la crise, à faire publier leurs communiqués par les journaux. À partir du 8 octobre, les journaux ont publié le texte intégral de chaque communiqué, aussitôt qu'il fut disponible. On a même reproduit les photocopies des pages couvertures de plusieurs communiqués, portant le dessin d'un patriote avec son fusil, sa tuque et sa pipe, accompagné des mots «Front de libération du Québec" et "Opération Libération". Mais on a également publié des photocopies des lettres des otages et celles-ci étaient généralement mises en relief par rapport aux communiqués, toujours au profit de l'élément de victimisation et de l'aspect pathétique de l'affaire. On constate ici dans quel dilemme sont placés les acteurs politiques radicaux quand ils ont recours à ce que Molotch et Lester (1974) appellent «disruptive access". Pour les acteurs marginalisés, commettre des actes qui dérangent la vie routinière constitue la seule façon d'accéder aux médias. Les gens qui possèdent le pouvoir ont facilement accès aux médias car ils constituent pour ces derniers des sources primaires. Mais ceux qui représentent une perspective opposée se doivent de capter leur attention par des actes extraordinaires. 
Cependant, même si les sources de définitions secondaires réussissent à infiltrer les médias, ceci ne leur assure pas nécessairement un traitement comparable aux définitions provenant des sources primaires. Prenons, par exemple, le traitement par les journaux du manifeste politique du F.L.Q. qui fut diffusé par Radio-Canada dans la soirée du 8 octobre, comme première concession du gouvernement fédéral. Notons que la demande originale des ravisseurs de $\mathbf{M}$. Cross voulait que le manifeste paraisse «intégralement en première page de tous les grands journaux du Québec" (communiqué $n^{\circ} 1$ de la cellule Libération). La première fois que ce manifeste fut publié dans les joumaux, c'est le 9 octobre, à la suite de sa diffusion par Radio-Canada la veille.

Seul le Journal de Montréal a consacré sa première page entière au texte, sous le titre: "Première concession: Radio-Canada a cédé!" Montréal-Matin annonce la publication du manifeste sur la première page: "INTÉGRAL: Le manifeste FLQ (voir page 10)". Le mot "INTÉGRAL" est imprimé en lettres rouges. La page dix est consacrée entièrement au texte du manifeste. Cependant, une grosse "Mise au point» signée "l'éditeur" met le lecteur en garde en ces termes:

On sait que nous réprouvons la violence, on sait que nous ne partageons pas la philosophie révolutionnaire des tenants FLQ. Mais s'il suffit d'un peu d'encre sur du papier pour que vive $M$. James Cross, alors nous sommes prêts à servir.

Nous avons ici un bon exemple de la façon dont le désir d'augmenter les tirages se dissimule sous une préoccupation humanitaire pour le sort de l'otage. Ceci est une conséquence directe de la stratégie des ravisseurs pour profiter de l'esprit de compétition en distribuant des copies de leurs documents aux différents médias.

La Presse publie le manifeste à la première page de la section des annonces classées (p. D1). Le reportage principal à la une signale au lecteur à quelle page se trouve le manifeste. Le texte du manifeste, qui occupe la moitié supérieure de la page, est précédé d'une explication: «la Presse pose ce geste dans un but humanitaire, espérant que cela contribuera à la libération de M. Cross». Quatre articles et une photo reliés à l'affaire Cross occupent la moitié inférieure de la page. Le Devoir du 9 octobre ne publie pas le texte intégral du manifeste mais présente un reportage sur son contenu à la dernière page de sa première section, dans un article intitulé «Le FLQ veut un Québec libre et uni, purgé de 'sa clique de requins voraces' " (le Devoir, 9 octobre: 16). L'article constitue un précis du texte rempli de citations directes mais expurgé de toutes les épithètes. C'est le seul article parmi les cinq de cette page qui porte sur la crise. 
The Gazette publie une traduction anglaise du texte intégral à la page 10, parmi d'autres articles portant sur la crise. À la une, le lecteur est renvoyé à cette page pour d'autres informations. Pourtant, la traduction anglaise du texte n'est pas tout à fait fidèle. L'exemple le plus flagrant est la référence au Premier ministre Trudeau. L'original dénonce «Trudeau la tapette", ce que The Gazette traduit par "chatterbox" (jacasse). Pour leur part, les journaux de l'après-midi-le Soleil et The Montreal Starne publient rien du manifeste. Le lendemain, samedi, le Soleil n'en publiera que les "points saillants» (le Soleil, 10 octobre: 3 ).

Or, on voit comment la présentation des mots du F.L.Q., même s'ils sont rapportés fidèlement et intégralement, ce qui n'est pas toujours le cas, peut saboter le message qu'ils comportent. On peut dire que le traitement du manifeste par le Journal de Montréal était le plus fidèle, mettant le texte intégral à la une et le laissant parler sans autres commentaires. Placer le texte parmi d'autres articles portant sur la crise, mais à l'intérieur du journal en diminue un peu l'impact. Ne placer qu'un résumé du texte parmi des articles non pertinents en réduit encore la portée. Accompagner le texte d'un désaveu est une façon explicite de délégitimer son message. Finalement, s'abstenir de publier le texte constitue la façon ultime de miner l'impact d'un message, c'est l'ignorer totalement.

\section{DEUX THÈMES CONTRADICTOIRES: LES NÉGOCIATIONS ET L'ENQUÊTE POLICIĖRE}

Le choix d'une manchette donne le ton à un reportage et présente une interprétation des événements qui en font l'objet. Les manchettes à la une des journaux, le vendredi suivant la diffusion du manifeste, donnent des exemples frappants des variantes possibles à cet égard. Le Devoir déclare: "Ottawa donne suite à deux exigences du FLQ" (Lépine, le Devoir, 9 octobre: 1). Dans son reportage, Normand Lépine prétend que «sans le reconnaitre publiquement, les autorités ont demandé à la police... de réduire au minimum les enquêtes et autres recherches qu'elle avait entreprises pour découvrir le repaire des ravisseurs...» La cessation de toutes les activités policières fut la deuxième demande minimale exigée par les ravisseurs de $\mathrm{M}$. Cross comme indice de bonne foi de la part des autorités.

À l'opposé, The Gazette déclare: «kidnap manhunt keyed to missing cabbie " ${ }^{5}$ (Collister et Radwanski, The Gazette, 9 octobre: 1). Il s'agit de la primeur d'une nouvelle sensationnelle: les deux reporters disparu.

5. L'enquête policière (littéralement "chasse à l'homme") vise un chauffeur de taxi 
prétendent que la police a réussi à identifier un premier suspect, un chauffeur de taxi montréalais qui est soupçonné d'avoir comploté l'enlèvement du consul et délégué commercial d'Israël, Moise Golan, en février 1970. Tandis que The Gazette ne dévoile pas son nom, le journal anglophone de l'après-midi, The Montreal Star, nomme Jacques Lanctôt (Poronovich et Conroy, The Montreal Star, 9 octobre: 2). Sa photo sera publiée à la une par The Gazette le lendemain. Dans la Presse, qui parait vers midi, la manchette déclare: «Le délai de minuit passe: rien $-\mathrm{Chez}$ les policiers, silence absolu.» Au sujet de l'enquête policière, on lit: «rien n'indique que les policiers aient cessé leurs recherches".

Pourquoi une telle divergence entre le Devoir et The Gazette? La différence linguistique constitue un indice. Dans une analyse de contenu des reportages de 22 quotidiens pendant la phase aiguë de la crise d'octobre, Siegel $(1974 ; 1983)$ a découvert que les thèmes sur lesquels porte l'emphase à la une, varient selon la langue du journal (voir Siegel, 1983: 213, tableau XI). Deux thèmes en particulier sont déterminants: "les négociations" pour la presse francophone et "l'enquête policière" (manhunt) pour la presse anglophone. Les thèmes auxiliaires sont aussi différents pour chaque groupe linguistique. Les francophones traitent du gouvernement fédéral et du gouvernement du Québec aux $2^{\mathrm{e}}$ et $3^{\mathrm{e}}$ rangs, alors que les anglophones en traitent aux $6^{\mathrm{e}}$ et $10^{\mathrm{e}}$ rangs. La sécurité et les mesures de guerre intéressent surtout les anglophones; ils en traitent aux $4^{\mathrm{e}}$ et $7^{\mathrm{e}}$ rangs, alors que les francophones en traitent aux $7^{\mathrm{e}}$ et $11^{\mathrm{e}}$ rangs.

On voit dans ces résultats deux modèles différents pour gérer la crise: pour les francophones, les négociations entre les gouvernements et les ravisseurs, et pour les anglophones, l'action policière, avec l'appui de l'armée et des mesures de guerre. Or, le Devoir et The Gazette se situent aux deux pôles de cette tendance polarisante. Ce contraste entre les approches des deux journaux est évident tout au long de la période étudiée. Par exemple, deux jours auparavant, le 7 octobre, les deux journaux présentent les interprétations opposées de la première déclaration officielle de Mitchell Sharp concernant la position fédérale. Le Devoir (7 octobre: 1) titre: "Ottawa cherche à négocier avec le FLQ", mais précise: "tout en rejetant les conditions posées". À l'inverse, The Gazette ( 7 octobre: 1) déclare en manchette "Demandes des ravisseurs rejetées" (traduction) avec la nuance suivante: "le gouvernement à la recherche d'un compromis avec les terroristes" (traduction). On voit donc comment le Devoir met l'accent sur le concept de "négociation", tandis que The Gazette accentue le concept de "rejet" ou de "refus".

Il est clair que, sur le plan de la légitimation, ces deux thèmes portent des significations très différentes. Dans la mesure où les felquistes 
sont présentés comme partenaires égaux dans un dialogue avec les autorités, ils sont légitimés comme acteurs politiques. L’insistance sur les négociations entre les deux cellules felquistes et les deux paliers gouvernementaux renforce cette image politique du F.L.Q. Dans la mesure où les felquistes sont présentés comme les objets d'une enquête policière, leur motivation politique demeure au second plan derrière leur action violente. L'accentuation de l'enquête policière et du refus par le gouvernement de négocier renforce l'image criminelle du F.L.Q.

On trouve dans l'édition du 13 octobre du Devoir (p. 5) un excellent exemple de la manière dont la composition d'une page peut renforcer une politique éditoriale. Robert Bourassa a fait une déclaration dans la soirée du 11 , où il laisse entendre que les vraies négociations peuvent se produire si les garanties pour la libération des otages sont fixées préalablement. Lors d'une réunion le 12, le comité de rédaction du Devoir décide de présenter la déclaration de $\mathbf{M}$. Bourassa comme une offre à négocier. Or, le jour où plusieurs journaux ont publié le texte intégral du manifeste, le Devoir n'en publiait qu'un résumé (le 9 octobre). C'est le 13 que le journal reproduit le texte intégral, interposé sur la même page entre les textes intégraux des déclarations de Jérôme Choquette (l'offre finale aux ravisseurs de $M$. Cross, livrée avant le rapt de $M$. Laporte) et de Robert Bourassa (sa déclaration du l1). La manière dont les trois textes sont agencés donne l'impression de trois déclarations d'égale valeur qui s'insèrent dans un dialogue. De plus, l'œil du lecteur est attiré d'un texte à l'autre, vers le coin inférieur de la page, à gauche, où on peut líre un encart publicitaire du "Centre d'études des communications" annonçant un programme d'activités sur le "perfectionnement en relations humaines 1970-1971".

Pour sa part, The Gazette (13 octobre: 6) publie un bref éditorial non signé, intitulé "Opening the way", qui conseille au public patience et compréhension vis-à-vis de la position du gouvernement, même si l'idée de négocier avec le F.L.Q. "répugne à la plupart des Québécois» (traduction). Sur la mème page, un article de Arthur Blakely donne une analyse rétrospective de la commission d'enquête Mackenzie sur la sécurité (cf. Canada, 1969). Selon le rapport de cette Commission, publiée en 1969 , les extrémistes québécois constituent, avec le communisme international, la principále menace pour la sécurité canadienne. Blakely affirme que, selon la Commission, "ce serait de la folie douce d'ignorer la menace poscé par les extrémistes au sein du mouvement séparatiste à la sécurité du Canada" (traduction). Les termes anglais de Blakely, "the lunatic fringe of the Quebec separatist movement", renforcent l'image des membres du F.L.Q. comme celle de gens insensés, dépourvus de 
raison et, donc, illégitimes en tant quaacteurs politiques. Sur la page opposée à la page éditoriale, The Gazette publie aussi une version anglaise de l'éditorial de Jean-Paul Desbiens, traduisant "L'anarchie ou la démocratie" par "The dice are tossed, but loaded, favoring anarchism" (The Gazette, 13 octobre: 7$)^{6}$. Il faut noter à quel point ce titre renforce l'image d'un F.L.Q. menaçant, avec comme message implicite: nos confrères français sont d'accord avec nous.

Les joumaux de langue française et de langue anglaise traitent différemment le thème de l'enquête policière autant que celui des négociations. Ceci apparaît surtout dans la couverture des événements auxiliaires qui concernent d'autres personnes que les otages, les ravisseurs et les autorités. Par exemple, seuls les deux journaux de langue anglaise ont rapporté le procès de Claude Morency, qui a débuté le 9 octobre (Levinson, The Gazette, 10 octobre: 10; Nebbs, The Montreal Star, 10 octobre: $3)^{7}$. Lors du témoignage d'un officier de l'escouade antiterroriste de Montréal, on découvre que le texte du communiqué saisi dans l'affaire Morency-Marcil-Lanctôt pose presque les mêmes demandes que celles des ravisseurs de James Cross, et qu'il contient un texte de quatre pages identique au manifeste lu sur les ondes de Radio-Canada jeudi soir, le 8 octobre.

En revanche, seuls les journaux de langue française font état de l'arrestation de Louis Fournier, reporter à CKAC, nommé par les ravisseurs de M. Cross comme observateur le 9 octobre $^{8}$. Son arrestation, pour homicide et vol à main armée à une caisse populaire, fut basée sur un mandat verbal. Elle servit de prétexte pour éloigner $M$. Fournier de son appartement afin de permettre une perquisition qui a effectivement dévoilé l'existence des exemplaires des trois premiers communiqués des ravisseurs de $M$. Cross et le manifeste du F.L.Q., textes que M. Fournier a reçus en tant que reporter à CKAC. Cette arrestation est un excellent exemple des illégalismes policiers qui sont devenus de plus en plus fréquents devant les difficultés éprouvées par la police à maintenir son enquête à l'intérieur des limites imposées par le due process.

Bien que ces deux événements soient reliés au thème de "l'enquête", ils ont un impact très différent. L'affaire Morency identifie ceux qui subissent une poursuite criminelle à ceux qui ont fait diffuser un

6. Les dés sont lancés, mais pipés en faveur de l'anarchie.

7. Morency est accusé, avec Pierre Marcil et François Lanctôt, le frère cadet de Jacques, un des ravisseurs de James Cross, de comploter l'enlèvement du consul américain, Harrison Burgess, en juin 1970.

8. Le Devoir, 10 octobre: 2; le Journal de Montréal, 10 octobre: 3; Cellier, MontréalMatin, 10 octobre: 3; Zolty, la Presse, 10 octobre: A2; Gagnon, La Presse, 10 octobre: A8. 
manifeste politique. Le fait d'en parler équivaut à assimiler le criminel et le politique. Traiter un acte politique comme un acte criminel le rend illégitime. À l'opposé, l'affaire Fournier remet en question la légitimité de l'enquête policière elle-même et, par inférence, les autorités gouvernementales. De plus, Foumier fut nommé par les ravisseurs comme observateur pour s'assurer de la mise à exécution de leurs demandes finales. Il est donc impliqué dans le processus de négociation, thème favori de la presse francophone. De la même façon, les silences réciproques renforcent les images divergentes de la légitimité des acteurs felquistes.

\section{LES NÉGOCIATIONS ET L'AMORCE D'UNE SYMÉTRIE DES IMAGES}

La période de trois jours du 12 au 14 octobre marque un tournant dans l'évolution de la crise, ce qui transparait dans le discours public. L'attention s'est déplacée du sort des otages vers le statut des négociations entre le gouvernement Bourassa et les ravisseurs. Dès le 12 octobre, à travers les thèmes et les voix traditionnels, tels que les otages, les paroles des autorités, l'enquête policière, et à travers les éléments suscitant la peur et la menace, tels que les appels à la bombe et les demandes de protection des hommes d'affaires, on remarque les indices d'un élargissement du focus. Par exemple, tandis que tous les journaux rapportent les paroles de M. Bourassa et de M. Choquette, deux d'entre eux mentionnent aussi que Paul Cliche, président du FRAP, l'opposition municipale dans la campagne électorale de Montréal, s'est déclaré en faveur des objectifs du F.L.Q. (Robitaille, la Presse, 12 octobre: B1; MontréalMatin, 12 octobre: 30 ). Certes, la nouvelle est enterrée à la page 30 dans le Montréal-Matin et totalement ignorée dans les autres quotidiens. Cependant, dans la Presse, un reportage assez complet sur l'assemblée du FRAP, accompagné d'une photo de Cliche, occupe la même page que deux articles sur Jean Drapeau et Lucien Saulnier, avec leurs photos. De plus, à la page B2, une grande photo de Me Choquette fait le pendant à celle de Robert Lemieux, l'avocat qui a défendu plusieurs felquistes et qui deviendra négociateur pour les deux cellules, et la page est annoncée à la une sous le titre "L'affrontement Choquette-Lemieux". À la page $B 8$, sous l'en-tete, "les hommes qui sont l'enjeu de l'escalade", on retrouve, bien entendu, les photos familières de MM. Laporte et Cross, mais aussi, celles des seize prisonniers felquistes. En-dessous de chaque photo des détenus, figure une brève description du dossier criminel de l'individu. En dessous des photos des otages on peut lire: «la vie de ces deux hommes..." et en dessous de l'ensemble des photos des détenus, en guise de réplique, la phrase «... contre la liberté de ceux-ci». 
Dès ce jour-là, on constate une sorte de symétrie dans le reportage, l'amorce d'un redressement du déséquilibre, le début d'une prise de conscience des deux partis de l'affaire. Même The Montreal Star, le plus modéré des deux journaux anglophones, a publié une critique acerbe de la ligne dure d'Ottawa signée par James Eayrs, professeur des relations internationales à l'Université de Toronto, et recommandant la libération des détenus afin d'épargner les otages ( 12 octobre: 5 ). Il ne s'agit que d'indices, de signes avant-coureurs, de symptômes. Les autorités, l'enquête, les victimes, la menace restent les thèmes privilégiés du reportage, exception faite des documents du F.L.Q.

C'est le 13 octobre que, pour la première fois, la majorité des photos à la une des journaux portent sur autre chose que les otages. Seuls The Gazette et le Soleil publient à la une des photos des membres de leurs familles. Sur les autres photos, figurent des militaires (camions ou soldats) à Ottawa, le représentant du gouvernement Bourassa dans les négociations, $M$. Trudeau et le frère de Robert Lemieux qui attend la libération de ce dernier. The Gazette, pour sa part, consacre sa deuxième page à l'enquête policière et aux demandes croissantes de protection. Trois des huit colonnes sont consacrées aux sept hommes impliqués dans l'affaire: M. Trudeau, M. Bourassa, Me Choquette, M. Sharp, Maurice St-Pierre (directeur de la Sûreté du Québec), Marcel St-Aubin (directeur de la police de Montréal) et Roland Jodoin (chef de l'escouade antiterroriste à Montréal et coordonnateur de l'escouade conjointe avec la SQ et la Gendarmerie royale du Canada). Pour chaque homme, une photo accompagnée de quelques paragraphes résumant sa carrière professionnelle; pour Robert Lemieux, le négociateur du F.L.Q., seulement un petit article sur le fait qu'il demeure toujours en prison. Cette page est l'exemple par excellence de la manière dont un reportage peut renforcer l'image des autorités en place.

Tout à fait à l'opposé, le Devoir consacre un article séparé à la déclaration du Parti québécois émise le 12 et appuyant "la démarche adoptée par le Premier ministre Robert Bourassa». Pourle Devoir, cette "démarche" signifie que $\mathrm{M}$. Bourassa est prêt à libérer les prisonniers politiques (le Devoir, 13 octobre: 1,6). D'autres journaux traitent cette nouvelle à l'intérieur de leurs éditions dans des articles séparés (la Presse, 13 octobre: A2; le Journal de Montréal, 13 octobre: 6; MontréalMatin, 13 octobre: 4; le Soleil, 13 octobre: 46). Remarquons que les journaux anglophones ne le font pas, à l'exception d'une petite mention de l'interprétation péquiste, dans l'article principal du Montreal Star (Poronovich, Nebbs et Dubois, 13 octobre: 1). Seul, le Devoir rapporte à la une (13 octobre: 1,6) la réaction du Parti communiste du Québec. 
Montréal-Matin (13 octobre: 20) enterre la nouvelle à l'intérieur de son édition. Silence chez tous les autres journaux. Les deux mêmes journaux mentionnent la réaction de Reggie Chartrand des Chevaliers de l'indépendance - l'aile droite du mouvement indépendantiste (le Devoir, 13 octobre: 6; Montréal-Matin, 13 octobre: 12). Seuls le Montréal-Matin (13 octobre: 20) et le Journal de Montréal (13 octobre: 6) rapportent que le syndicat des professeurs de l'Université de Montréal dénonce l'arrestation d'un de ses membres, Charles Prévost.

Ces petits silences permettent de constater très subtilement mais clairement la concentration de l'attention sur les sources primaires. Que le F.L.Q. et Robert Lemieux aient acquis, dans les reportages, un statut comparable à celui des hommes publiques signifie que l'amorce des négociations a accru leur légitimation. Il est intéressant de noter, à cet égard, que, depuis le 13 octobre, le Soleil utilise l'en-tête "L'AFFRONTEMENT FLQ - GOUVERNEMENT" pour les pages traitant des négociations et des événements à Ottawa (le Soleil, 13 octobre: 3; 14 octobre: 19). Cet en-tête met le F.L.Q. et les gouvernements sur un pied d'égalité.

Le 14 octobre, toutes les manchettes rapportent la rupture des négociations qui a eu lieu la veille. Les journaux de l'après-midi mettent l'accent sur la reprise des négociations suite à une réponse des deux cellules felquistes. Chose frappante: aucune photo des otages, ni de leurs résidences, ni de leur entourage, ne figurent à la une, le 14 octobre. De plus, aucune photo des victimes n'apparait nulle part. On voit Robert Lemieux, ses conférences de presse, les hommes politiques, les soldats, les arrestations. Mais les otages ont disparu. La situation est la même le 15 octobre: absence frappante de commentaires sur les otages, surtout à la une. Seul The Gazette publie un article sur leurs familles avec une photo de chaque otage. Mais sur la même page, des photos de trois des "seize" - René Lévesque, Claude Ryan et Louis Laberge - accompagnent un article sur leur déclaration. Le pattern de la couverture s'est déplacé du sort des otages vers un débat plus généralisé sur les négociations. Pour beaucoup de gens, le véritable enjeu est devenu le droit pour le Québec de poursuivre seul les négociations, indépendamment d'Ottawa'.

9. Les seize, dans leur déclaration, affirment: «L'affaire Cross-Laporte est avant tout un drame québécois». C'est contre eux que $M$. Robarts a déclaré "la guerre générale", insistant sur le fait que les enlèvements représentent un problème national, pas simplement québécois. 


\section{LA RIPOSTE OFFICIELLE: UN CHANGEMENT ABRUPT DU MODĖLE}

Avec l'arrivée de l'armée au Québec, le 15 octobre, la couverture se jette sur la présence des soldats à Montréal et à Québec. De plus, à Ottawa, M. Trudeau annonce aux Communes l'annulation de son voyage en Union soviétique prévu pour le 19 octobre. Les deux journaux de l'après-midi rapportent la nouvelle à la une. Dans le Soleil, sur cinq articles à la une portant sur l'affaire Cross-Laporte, le plus court traite des négociations et le plus long est un reportage choc intitulé «Bourassa retarde à la limite l'instauration de l'État policier». Donc, entre le matin et l'après-midi, l'accent du reportage se modifie brusquement.

Cette tendance s'accentue encore le 16. Sur douze photos à la une, dix montrent l'armée: les soldats à Montréal ou à Québec, les convois de camions, les hélicoptères. Dans The Gazette, la photo d'une fillette avec une sucette et un soldat en arrière-plan porte la légende: «Évocateur d'une scène d'Europe en temps de guerre" (traduction). Les deux autres, toutes deux dans le Soleil, dépeignent une perquisition policière dans une librairie progressiste de Québec. L'armée, la police et les deux gouvernements font les manchettes à la une. Cinq manchettes parlent du F.L.Q. de façon péjorative: "Bourassa dit NON au FLQ" (la Presse), "Ottawa outlaws FLQ" (The Montreal Star), «l'étau se resserre sur les terroristes» (le Journal de Montréal). On constate ici que le F.L.Q. vient de perdre sa légitimité fraîchement acquise.

Aucune manchette ne fait allusion aux négociations qui se sont terminées la veille quand Robert Lemieux a rejeté l'offre finale de Robert Bourassa. Toujours rien à la une sur les otages, sauf une manchette du Montréal-Matin qui se lit ainsi: "On saurait où est Laporte" " ${ }^{10}$. Une seule manchette (The Gazette) parle de "l'avocat du FLQ" (traduction) avec son nom en sous-titre seulement. La loi sur des mesures de guerre, la vague des arrestations et perquisitions, l'arrivée de l'armée à Montréal et à Québec et la mise hors-la-loi du F.L.Q. constituent les principaux thèmes des reportages.

Un aspect frappant de la couverture du 16 octobre est la résurrection des thèmes de menace et de danger. La spéculation délirante sur le sort des otages et sur les effectifs supposés du F.L.Q. en sont des exemples. Le Montréal-Matin (Cellier et Paré, 16 octobre: 2) parle d'une descente imminente dans le lieu de séquestration de Pierre Laporte. The Gazette fait état d'un rapport secret de la GRC affirmant que le F.L.Q. a 22

10. Il s'agit d'une conférence de presse où Robert Lemieux affïrme que la police connaît le lieu où $\mathrm{M}$. Laporte est sequestré, ce qui s'est avéré faux par la suite. 
cellules actives et plus de 2000 sympathisants (Windeyer, 16 octobre: 8). L'apparition de tels reportages juste après la fin des négociations et la mise en action de la riposte officielle n'est pas une coïncidence. Les thèmes de la peur et de la victimisation confirment l'image criminelle du F.L.Q. l'assimilant à un groupe capable de monter une insurrection. Qui plus est, on voit la réapparition des reportages sur les vols d'armes et de dynamite, même s'ils ne sont pas reliés avec le F.L.Q. Par exemple, le Devoir (16 octobre: 2) rapporte "en bref" le vol des mitraillettes et des culasses de fusils militaires dans un manège de la milice en ColombieBritannique. Ce même vol avait été mentionné à la Chambre des Communes, le jour précédent.

La couverture du 17 octobre reflète d'une façon frappante le changement abrupt dans le discours public survenu la veille. L'attention est exclusivement tournée vers la version officielle des événements, les explications des autorités pour l'invocation des mesures de guerre, et le débat entourant cette décision. Tandis qu'auparavant, les communiqués felquistes et les commentaires de Robert Lemieux remplissaient les journaux, maintenant, ce sont les textes issus des leaders gouvernementaux ou de la loi elle-même. Ensemble, ils constituent une litanie de déclarations officielles qui réitèrent l'autorité du gouvernement: trois textes légaux, trois lettres officielles, deux communiqués gouvernementaux, et deux discours du Premier ministre du Canada. De plus, les journaux sont truffés de reportages sur le flot du discours parlementaire et sur les conférences de presse des ministres fédéraux et provinciaux ${ }^{11}$.

La majorité des manchettes et des articles à la une traitent des hommes politiques et de leurs déclarations, ainsi que des arrestations. Quand on mentionne le F.L.Q., c'est pour dire qu'il est maintenant hors-Ia-loi, qu'il constitue un grand danger ou qu'il est maintenant complètement muet. Si on parle des otages, c'est pour s'inquiéter de n'en avoir aucune nouvelle depuis des jours. Plusieurs journaux rapportent la réplique de René Lévesque à l'action gouvernementale, mais la plupart insistent surtout sur son appel aux ravisseurs pour la libération des otages plutôt que sur ses critiques acerbes des gouvernements. Trois d'entre eux, le Journal de Montréal, le Devoir et le Soleil, mentionnent à la une sa déclaration selon laquelle le Québec n'a plus de gouvernement. Seuls le Devoir et The Montreal Star présentent, dans leurs reportages à la une, les critiques des chefs de l'opposition fédérale ${ }^{12}$.

11. Pour une analyse détaillée de tous ces textes et discours, voir Crelinsten, 1985, ch. $18,19,21$.

12. La manchette dans le Devoir se lit: «Le gouvernement est allé trop loin» (Stanfield et Douglas). 
Des quinze photos à la une, quatre présentent des soldats, six, des hommes politiques ${ }^{13}$, deux, des arrestations (dont celle de Pauline Julien dans le Journal de Montréal), deux, des otages (seulement dans la Presse) et une seule, des étudiants manifestant en faveur du F.L.Q. (le Soleil) ${ }^{14}$. C'est peut-être l'en-tête du Soleil qui représente le mieux le pattern de communication qui s'est développé suite à l'invocation de la Loi sur les mesures de guerre. La manchette principale déclare sur deux lignes: "Trudeau tente de rassurer la nation». Au-dessus, un petit titre: «Où sont les otages?» En-dessous, un sous-titre: «Mutisme complet du Front de libération du Québec" $" 15$.

Le sort des otages, surtout la mort de Pierre Laporte, et l'activité policière et militaire occupent exclusivement les reportages du 18 sur la crise. Dans le même esprit, le 19 octobre, jour où le Parlement canadien approuva par une vote de 190 contre 16 l'adoption des mesures d'urgence, la couverture à la une se consacre presque exclusivement aux sujets suivants: M. Laporte et le deuil national, une lettre de M. Cross diffusée à la radio le jour précédent ${ }^{16}$, l'enquête policière, et les déclarations des hommes politiques. Cinq des sept journaux ont publié à la une les photos de Paul Rose et de Marc Carbonneau, deux des ravisseurs, avec des reportages sur les mandats d'arrestation émis à leurs noms. Les deux autres - le Devoir et le Soleil - ont publié ces photos en deuxième page. The Gazette a aussi publié la photo de Jacques Lanctôt, pour une troisième fois. À l'exception de la Presse qui utilise des photos ordinaires, tous les journaux publient les photos d'identification judiciaire de MM. Rose et Carbonneau qui accompagnaient leurs mandats d'arrestation. Tandis que la plupart des journaux se sont limités aux clichés de face, The Gazette a publié aussi les profils, avec le signalement de chaque suspect et, pour Paul Rose, le numéro matricule reçu à la prison de Montréal en 1968, quand il fut arrêté lors d'une manifestation.

Avec ces photos, le cercle se referme et l'image des felquistes retrouve son caractère criminel. Ceux qui avaient acquis une image

13. Trudeau: 2, Marchand: I, Bourassa: I, Stanfield: 1, Douglas: 1.

14. Cette photo est accompagnée d'une autre qui dépeint l'arrestation d'un jeune homme.

15. Il faut noter que ce mutisme du F.L.Q. résulte en partie des craintes des médias d'information. De fait, les ravisseurs de James Cross émettent, le 17, un dixième communiqué, accompagné d'une lettre manuscrite de M. Cross. CKLM fut averti, mais le poste décide d'appeler la police plutôt que d'aller chercher le communiqué et de risquer une poursuite criminelle.

16. Il s'agit d'une deuxième lettre, envoyée le 18, et que CKLM a ramassée et a diffusée une fois, avant que la police $n$ "interdise toute émission additionnelle. Aucune photocopie n'est faite de cette lettre (voir Crelinsten, 1985: 468-472). 
transitoire d'acteurs politiques poursuivant des buts légitimes redeviennent, aux yeux des gens, de vulgaires criminels. Les images de la dépouille de $\mathbf{M}$. Laporte, de sa femme et de sa mère en deuil, des leaders politiques rendant hommage à sa dépouille, du lieu de sa séquestration, et celles particulièrement lugubres de son cadavre dans le coffre d'une voiture, identifient le F.L.Q. à une bande de meurtriers.

\section{CONCLUSION: APERÇU D'UNE AUTRE RÉALITÉ}

Le 11 octobre, l'hebdomadaire indépendantiste Québec-Presse déclare, dans un éditorial intitulé «Le FLQ et nous»:

Le FLQ a réussi à lui parler, à cette population [du Québec], comme jamais encore auparavant, en une seule semaine. L'action du FLQ aura été une sorte de cours de politisation par immersion totale. Une sorte d'école Berlitz de la politique. Ainsi, le FLQ a atteint un objectif majeur: parler, dans ses mots, au monde. Et retenir l'attention de toute la population québécoise sur la situation des Québécois.

(Québec-Presse, 11 octobre: A40)

Il est vrai que les ravisseurs de James Cross ont réussi à parler dans leurs mots, après avoir gagné la lutte pour faire publier leurs communiqués et leur manifeste. Il est aussi vrai qu'ils ont monopolisé l'attention de la population pendant une semaine. La couverture de presse reflète ces deux tendances. Donc, on peut dire que la cellule felquiste est parvenue à attirer l'attention et à être reconnue.

Quant à l'obtention de la légitimation, le problème est plus complexe. Québec-Presse parle d'une «école Berlitz de la politique». Cependant, c'est une chose de se faire comprendre dans ses propres mots et une autre de gagner l'approbation générale. Le F.L.Q. a certainement parlé, mais il a aussi enlevé un individu par la force. Presque toute l'attention de la presse fut centrée sur cet aspect de l'action felquiste. De fait, pendant la première semaine, la couverture de presse, à part celle du Devoir ${ }^{17}$, fut remarquablement pauvre en analyses du contexte social ou historique de la crise.

Cependant, l'impact sur la population du manifeste - les parolesmêmes du F.L.Q. - est beaucoup plus évident à travers un autre médium, la radio, surtout dans les lignes ouvertes des postes montréalais de langue française. Bien que la plupart des personnes qui s'expriment condamnent l'action felquiste, plus de $50 \%$ d'entre elles se déclarent en faveur de l'esprit du manifeste (Raboy, 1981: 62). Mais prenons garde. Si

17. Le lendemain même de l'enlèvement, Claude Ryan a écrit un éditorial, intitulé "La violence entre dans une nouvelle phase", qui reste une des meilleures analyses du terrorisme dans le contexte de la guerilla urbaine, et des risques qu'il pose pour une démocratie (Ryan, le Devoir, 6 octobre: 8). Mais de telles réflexions furent plus que rares avant l'enlèvement de Pierre Laporte. 
les paroles se sont méritées la sympathie ${ }^{18}$, les actes, par contre, ont attiré l'attention et la désapprobation, ce qui s'est reflété dans la couverture de presse. Nous avons vu que les quotidiens ont concentré leurs reportages sur les thèmes traditionnellement traités lors d'un incident de violence politique: la victime, la menace, le passage du temps, et la réaction des autorités comme sources légitimées, et donc primaires, de tout commentaire.

Cependant, les communiqués du F.L.Q. - les paroles - sont devenus une monnaie précieuse pour la presse: une source d'information sur les demandes, les délais, et l'état des otages. Leurs mots ont donc pénétré les reportages en tant que produits de consommation, parce qu'ils attirent les lecteurs. Du même coup, on renforce les thèmes de peur et de menace. Ici on voit les vrais enjeux du travail des médias. L'idéologie de "l'intérêt public" sert de justification à une couverture sensationnelle qui a pour but d'alimenter l'intérêt du public. Le "droit de savoir" se métamorphose en droit de s'accrocher aux détails morbides aux dépens des analyses contextuelles. C'est ainsi que Paul Gros d'Aillons, éditorialiste du Montréal-Matin, défend le rôle des médias dans la crise en s'appuyant sur le principe selon lequel on promet de «donner toutes les nouvelles, tous les commentaires, tous les détails" (Gros d'Aillons, Montréal-Matin, 14 octobre: 8). Il est ironique que, dans le même éditorial, l'auteur affirme que "les tirages des journaux atteignent des records incroyables», tellement la demande est grande pour de tels propos.

La pénétration des mots felquistes dans la couverture de presse a eu deux résultats. On a appris le point de vue du F.L.Q. à travers ses propres mots. De plus, une sorte de dialogue s'est développé au cours de la première semaine, entre les ravisseurs et les gouvernements. Le F.L.Q. a gagné encore plus de légitimité sur le plan symbolique du dialogue que par la simple publication de son manifeste. Cependant, cette légitimité est limitée par l'attention accordée à l'acte violent et à la menace pesant sur la victime et par l'insistance sur l'enquête policière et les déclarations des sources primaires. À mesure que la presse écrite a dégagé ces éléments, elle a freiné le processus de légitimation des felquistes et, du même coup, elle a renforcé l'image criminelle et terroriste telle que promulguée par les autorités. La tendance à présenter l'acte hors de son contexte politique a eu le même effet. Ceci fut plus évident dans la couverture en anglais, mais ce fut aussi vrai dans les quotidiens de langue française.

18. Effectivement, dans le sillage de la crise, il y eut des initiatives fédérales et provinciales sur le pian de création d'emplois (fédéral) et de la réforme sociale (provincial) (voir Raboy, 1983: 66; Dion, 1985: 196). Selon Raboy, ces tentatives eurent pour effet principal la cooptation des forces progressistes et populaires dans la province, par l'appareil d'Êtat. L'ironie c'est que, dans une perspective radicale, il s'agit de cooptation, mais dans une perspective d'État, il s'agit de prévention sociale. Cette contradiction nous amène trop loin de notre sujet, mais il suffit à noter que ces initiatives «post-crise» de la part de l'État constituent les indices que les deux paliers gouvemementaux ont appris quelque chose, eux aussi, à l'école Berlitz du F.L.Q. 
La mise au second plan du sort des otages au profit d'un débat plus généralisé sur les enjeux politiques, dans la deuxième semaine de la crise, a accéléré le processus de légitimation du F.L.Q. en court-circuitant l'image criminelle qui est soutenue par le thème de victimisation. Cette légitimation transparaît d'une manière subtile, dans la présentation symétrique des photos et des déclarations des interlocuteurs. Jamais on n'a cessé de présenter les sources primaires traditionnelles. Cependant, une sorte d'équilibre entre sources officielles et sources oppositionnelles a modifié pour quelques jours le modèle traditionnel. On a atteint une symétrie précaire entre la position gouvernementale et la position felquiste. En temps ordinaire, la norme est asymétrique, la position dissidente restant invisible ou au second plan par rapport à la position officielle. Après l'invocation des mesures de guerre et l'irruption du discours officiel dans la presse, la symétrie établie au cours des jours précédents s'évanouit et le déséquilibre normal réapparaît en force. De par ces transitions - asymétrie, symétrie, asymétrie - on note un dérangement transitoire dans la reproduction de l'ordre que représente la couverture de presse en temps ordinaire.

Or, il m'apparaît que les temps de crise peuvent aider le chercheur à découvrir des modèles qui, en temps ordinaire, échapperaient à son attention à cause de leur banalité. Criminaliser un acteur politique parce qu'il utilise la violence c'est lui enlever sa légitimité. En temps normal, le reportage qui met l'accent sur la violence renforce le modèle pénal. En temps de crise, nous avons vu l'aspect violent disparaitre, laissant voir, pour une brève période, l'image d'un acteur politique dissident mais légitime, une réalité autre que celle quotidiennement présentée par nos médias. Du même coup, on découvre la fonction légitimante des médias, qui, en temps ordinaire, mettent l'accent sur les sources officielles et sur la violence pour la violence. Loin d'être une source sûre de légitimation pour les acteurs dissidents et violents, la couverture par la presse renforce de façon systématique l'image officielle de criminalité promulguée par les autorités. $\mathrm{Si}$ on peut percevoir une certaine légitimation dans les images symétriques des felquistes et des autorités publiées pendant la crise d'octobre, il s'agit d'une exception à la règle générale. De fait, la nature exceptionnelle de cette légitimation chimérique découle du fait qu'elle ne surgit qu'en temps de crise aiguë. Du même coup, la règle générale qui veut que la couverture de presse renforce de façon systématique les perspectives officielles devient tout à fait évidente au moment où justement elle échoue au plus fort de la crise. 


\section{BIBLIOGRAPHIE}

ALLEN, F.A. (1974), The Crime of Politics: Political Dimensions of Criminal Justice, Cambridge, Mass., Harvard University Press.

CANADA (1969), Rapport de la Commission royale d'enquête sur la sécurité (version abrégée), Ottawa, L'imprimeur de la reine.

COHEN, S. and J. YOUNG (édit.) (1981), The Manufacture of News: Social Problems, Deviance and the Mass Media, Revised Edition, London, Constable.

CRELINSTEN, R.D. (1985), "Limits to Criminal Justice in the Control of Insurgent Political Violence: A Case Study of the October Crisis of 1970", thèse de doctorat inédite, Université de Montréal.

DION, G. (1985), Une tornade de 60 jours: la crise d'octobre 1970 à la Chambre des Communes, Hull, Éditions Asticou.

HAGGART, R. and A.E. GOLDEN (1979), Rumours of War, Toronto, James Lorimer (L'édition originale publiée en 1971).

HALL, S., C. CRITCHER, T. JEFFERSON, J. CLARKE and B. ROBERTS (1978), Policing the Crisis: Mugging, the State, and Law and Order, London, Macmillan.

HALLORAN, J.D. (1981), "Mass Communication: Symptom or Cause of Violence", in Violence and Its Causes, Paris, UNESCO, p. 125-140.

KELLY. M.J. and T.H. MITCHELL (1981), "Transnational Terrorism and the Western Elite Press", Political Communication and Persuasion, vol, 1, n 3, p. 269-296.

MOLOTCH, H.L. and M. LESTER (1974), "News as Purposive Behavior: On the Strategic Use of Routine Events, Accidents, and Scandals", American Sociological Review, vol. 39, p. 101-112.

MURDOCK, G. (1981), "Political Deviance: the Press Presentation of a Militant Mass Demonstration", in S. Cohen and J. Young (édit.), The Manufacture of News, London. Constable, p. 206-225.

PROVENCHER. J. (1974), la Grunde peur d'octobre 70, Montréal, L'Aurore.

RABOY, M. (1983), Libérer la communication: Médias et mouvements sociaux au Québec, 1960-1980, Montréal, Nouvelle Optique.

SCHLESINGER, P.. G. MURDOCK and P. ELLIOT (1983), Televising 'Terrorism': Political Violence in Popular Culture, London, Comedia.

SCHMID, A.P. and J. DE GRAAF (1982), Violence as Communication: Insurgent Terrorism and the Western New's Media, Beverly Hills, Sage.

SIEGEL, A. (1974), "Canadian Newspaper Coverage of the FLQ Crisis: a Study of the Impact of the Press on Politicsn, thèse de doctorat inedite, McGill University.

SIEGEL, A. (1983), Politics and the Media in Canada, Toronto, McGraw-Hill Ryerson.

TUCHMAN, G. (1978), Making News: A Study in the Construction of Reality. New York, The Free Press. 